\title{
EL TRATAMIENTO DEL PRESENTE DE SUBJUNTIVO PARA OPINAR O VALORAR EN LOS MANUALES DE ESPAÑOL LENGUA EXTRANJERA
}

Laura Arroyo Martínez ${ }^{1}$

Universidad Rey Juan Carlos

Recibido 25/10/2020 Aceptado 30/11/2020

En el presente artículo se analiza el tratamiento del presente de subjuntivo en el valor de opinión o valoración dentro de los manuales de Español como lengua extranjera. Se ha seleccionado un corpus representativo de manuales y se ha analizado mediante un enfoque cuantitativo y cualitativo los siguientes aspectos: si este contenido gramatical se nombra de manera explícita en el índice de los manuales; si dentro de las unidades o lecciones donde se trabaja este contenido se aporta información teórica relevante para los estudiantes; si se desarrollan actividades que ayuden a los estudiantes a aprender e interiorizar esta estructura y a qué metodología pertenecen y, por último, qué tipo de diferencias existen entre los manuales.

5 This article analyzes the treatment of the present subjunctive in the value of opinion or valuation within the manuals of Spanish as a foreign language. A representative corpus of manuals has been selected and the following aspects have been analyzed using a quantitative and qualitative approach: if this grammatical content is explicitly named in the manual's index; if within the units or lessons where this content is worked on, relevant theoretical information is provided for students; If activities are developed that help students to learn and internalize this structure and to what methodology they belong and, finally, what kind of differences exist between the manuals.

DOI

https://doi.org/10.15366/didacticas2020.23.002

PALABRAS ClaVE

ELE; Gramática; Subjuntivo; Análisis de materiales. 


\section{INTRODUCCIÓN: LA IMPORTANCIA DE LA ADQUISICIÓN DEL PRESENTE DE SUBJUNTIVO EN ELE}

Todo docente y/o investigador en el ámbito de la Enseñanza del español como lengua extranjera (en adelante, ELE) es conocedor de la posición clave que ocupa, dentro de los contenidos gramaticales, el aprendizaje del presente de subjuntivo a partir del nivel B1 en los cursos de español. Como indica el Plan Curricular del Instituto Cervantes (en adelante, PCIC) si un discente ha alcanzado el nivel B1, en lo relativo a su conocimiento del presente de subjuntivo, debe conjugar adecuadamente los verbos regulares de las tres conjugaciones, las irregularidades heredadas del presente de indicativo, así como las irregularidades en el tema y en la raíz de los verbos estar, ser, dar e ir. En cuanto a los usos que debe dominar (denominados valores en el PCIC), resalta el empleo de oraciones independientes, orientado al presente o al futuro, entre las que se encuentran las siguientes construcciones: expresiones desiderativas, expresiones de duda, estilo indirecto en presente, construcciones impersonales que suponen juicio valorativo, subordinadas adverbiales temporales introducidas por antes de que, después de que y cuando (con valor de futuro), así como oraciones adverbiales finales introducidas con 'para que.'

Como se deduce del listado que se acaba de referenciar, estos contenidos son amplios cuantitativamente y complejos de abordar cualitativamente en el ámbito de la didáctica. Como bien ha indicado Ughetta Gouverneur, quien ha diseñado una propuesta de actividades desde el enfoque pragmático y cognitivo para trabajar el subjuntivo, nos encontramos ante "uno de los temas más ricos de interés en el ámbito de la enseñanza aprendizaje del español” (Ughetta Gouverneur: 2013, 84).

También se debe indicar que el aprendizaje del presente de subjuntivo es uno de los aspectos más problemáticos en el aprendizaje del español y, a la vez, uno de los más necesarios por el alto rendimiento que este tiempo posee en nuestra lengua. Es decir, nuestros aprendices necesitan emplearlo adecuadamente puesto que en español hay un número muy importantes de funciones comunicativas que seleccionan dicho tiempo verbal (desear, indicar duda o probabilidad, indicar acciones en el futuro etc.) Esta necesidad comunicativa hace que, cuando nuestros aprendices de español desean emplear el presente de subjuntivo y no se sienten seguros, opten por seleccionar otras expresiones que no emplearía con frecuencia un hablante nativo con el fin de esquivar el empleo erróneo de dicho contenido gramatical. ${ }^{2}$

\footnotetext{
1 Para un listado exhaustivo véase [Enlace].

2 Para quienes somos examinadores oficiales del DELE o hemos examinado en otros contextos no es difícil encontrar casos recurrentes en los que los candidatos o estudiantes producen expresiones del tipo: "Esta noche celebro una fiesta. ¿Te gustaría venir?" frente a otra expresión como "Quiero que vengas a la fiesta que celebro esta noche" y, en muchos casos, esta selección no es debida a que la primera marca una clara invitación y es mucho más formal desde el punto de vista pragmático, sino que permite al estudiante salvar el empleo del subjuntivo. Los estudiantes o candidatos intentan no emplearlo porque realmente les resulta costoso y no quieren cometer errores que puedan ser motivo de penalización. En muchos casos son los profesores o examinadores quienes deben formular preguntas que lle ven a los estudiantes o candidatos a un empleo obligatorio de dicho modo verbal.
} 
Por todo ello, es sencillo deducir que los especialistas en ELE han centrado una parte muy importante de sus esfuerzos en el diseño de gramáticas pedagógicas que faciliten el aprendizaje del subjuntivo ${ }^{3}$, con apuestas por la inclusión de nuevas metodologías como las basadas en las aportaciones de la lingüística cognitiva y la pragmática, que defienden que la diferencia entre la selección de indicativo frente a la del subjuntivo "no es lingüística, sino pragmática: según el contexto en que se interprete, el estatus del evento introducido por el subjuntivo será diferente. El modo verbal aporta una información esquemática, que debe completarse a través de la inferencia." (Amenós, Ahern y Escandell: 2019, $56)$.

Por consiguiente, desde este enfoque se puede demostrar que la explicación tradicional y estructuralista de la alternancia de modo no resulta provechosa para el aprendizaje de nuestros estudiantes. De esta insatisfacción de resultados también han surgido propuestas que afectan a la remodelación de la secuenciación de contenidos, como es el caso de la investigación presentada por Contreras y Ferreira, para quienes "cabe preguntarse si el subjuntivo en español podría ser más aprendible si antes de intentar enseñárselo pudiéramos determinar el grado de madurez lingüística de los estudiantes dentro de la secuencia de adquisición del ELE” (Contreras y Ferreira: 2013, 256).

Pese a esta incesante labor, los resultados en el aprendizaje del presente de este modo verbal todavía están lejos de ser óptimos puesto que, como ha señalado Jiehui Zu (2018: 94), "sigue destacando la dificultad de adquirirlo para los estudiantes extranjeros, la cual, se intensifica en las lenguas maternas de dichos estudiantes más distantes del español”. Prueba de ello es que el empleo del presente de subjuntivo sigue siendo central en el estudio del análisis de errores de los aprendices de español, como demuestran los estudios que se han publicado sobre dichos errores estudiantes finlandeses (Rodellas y Rodríguez, 2018), polacos (Castro Cruz, 2018), francófonos (Dida Nopogwo, 2019) o lituanos y letones (Llamas Sevilla, 2020), entre otros. Por consiguiente, por la relevancia pedagógica que presenta el aprendizaje del presente de subjuntivo en el aula ELE, considero necesario realizar un análisis que permita comprender cómo se aborda dicho contenido en manuales de referencia dentro de la gama de posibilidades que presenta el mundo editorial ELE.

Respecto al tema del presente estudio, no se puede dejar de resaltar la gran importancia que se debe aportar al análisis de materiales, campo de investigación esencial en ELE, para poder conocer en profundidad las propuestas didácticas existentes, así como sus posibles vías de explotación en la gran diversidad de contextos pedagógicos que encontramos actualmente en la variada enseñanza de nuestra lengua. Esta indagación en los manuales resulta esencial para los docentes puesto que, como es reconocido, el empleo de

3 Para abordar el aprendizaje del modo subjuntivo en contraste con la selección del modo indicativo en oraciones su bordinadas sustantivas supone de gran ayuda el artículo de Tordera Yllescas (2016) en el que se presentan unas re glas de fácil explicación didáctica para los discentes, así como una propuesta de actividades que presenta una gran rentabilidad. Nos referimos al artículo "A vueltas con el subjuntivo en la clase de español como lengua extranjera: subjuntivo y oraciones sustantivas" (2016). 
manuales no es algo secundario en la praxis docente, sino que se presenta como un recurso de primer nivel puesto que el profesor "aprovecha el material que pueda haber en el manual y realiza variaciones sobre los contenidos y actividades que presenta" (De Santiago y Fernández: 2017, 684) Es decir, el manual se convierte en muchas ocasiones en un gran apoyo para el docente y es, por esta razón, por la que debe estar muy familiarizado con el mismo.

\section{OBJETIVOS Y METODOLOGÍA}

Como se ha explicado en la introducción de este artículo, la enseñanza del presente de subjuntivo se encuentra de manera recurrente en las programaciones de nivel intermedio de los diversos cursos de ELE. Del mismo modo, como se ha indicado, la presente investigación se concentra en el análisis de materiales ELE y, muy concretamente, en el tratamiento del presente de subjuntivo en los mismos. Para conseguir llevar a cabo de manera rigurosa nuestro objetivo principal hemos seleccionado diez manuales de español como lengua extranjera que cumplieran los siguientes criterios: que estuvieran destinados a estudiantes jóvenes o adultos, que fueran del nivel B1 (al ser este el nivel en el que se introducen este tiempo verbal), que fueran de distintas editoriales y autores y que hubieran tenido una importante difusión, es decir, que fueran conocidos por un número importante de docentes y, por último, que puedan ser empleados por estudiantes de distintas nacionalidades, es decir, que no fueran manuales especializados en estudiantes de una determinada segunda lengua para que el público potencial de empleo no se encuentre restringido.

De esta manera, conseguimos aportar una muestra representativa que permita alcanzar conclusiones sólidas.

La metodología de esta investigación se va a sustentar en el esquema presentado por Artusi en un artículo publicado este mismo año en esta misma revista ${ }^{4}$. Con el apoyo en esta investigación, que nos sirve de referente, en el presente artículo se va a llevar a cabo una investigación cualitativa y cuantitativa de los manuales seleccionados mediante la que vamos a responder a las siguientes preguntas de investigación, relacionadas con la presentación de los contenidos y las actividades que dichos materiales presentan para conseguir el aprendizaje del presente de subjuntivo en construcciones cuya función comunicativa sea dar una opinión o hacer un juicio de valor:

1. ¿Se expone de manera explícita en los índices de los manuales la presentación del presente de subjuntivo en construcciones cuya función comunicativa sea expresar la opinión dentro de la unidad o unidades didácticas correspondientes en el manual o, por el contrario, esta información no se aporta de manera explícita?

4 Nos referimos al valioso artículo titulado "El tratamiento de los phrasal verbs en los manuales de inglés lengua extranjera." (Artusi: 2020) 
2. Dentro del manual, ¿existen apartados teóricos en los que se facilitan explicaciones lingüísticas y no lingüísticas sobre el empleo del presente de subjuntivo en dichas construcciones? Si la respuesta es afirmativa, ¿qué tipo de explicaciones se aportan?

3. ¿Se presentan información del presente de subjuntivo para formular opiniones o juicios de manera independiente o este tiempo verbal se estudia sistemáticamente en contraste con el presente de indicativo?

4. Si se presentan actividades para la ejercitación del presente de subjuntivo en la función referida, ¿a qué enfoque metodológico atienden y en qué consisten?

5. ¿Qué similitudes y que diferencias se encuentran en los manuales analizados en lo que respecta al tratamiento del presente de subjuntivo para expresar la opinión?

Uno de los elementos de mayor utilidad para los docentes a la hora de seleccionar y emplear un determinado manual dentro del aula es conocer de qué tipo son las actividades que presenta, puesto que la tipología de las mismas condiciona notablemente las posibilidades de conseguir los objetivos pedagógicos que se ha planteado en el diseño del curso. En el presente trabajo, para llevar a cabo el análisis de las actividades que se presentan sobre el presente de subjuntivo para dar la opinión se realiza una clasificación de la tipología de las mismas, atendiendo a las siguientes agrupaciones:

1. "Ejercicio mecánico", que en el caso del subjuntivo se centra en el refuerzo del aprendizaje de la morfología. Es decir, en este tipo de actividad no se requiere para el alumno la comprensión del uso de este tiempo verbal, ni de la necesidad de identificar la selección entre el presente de indicativo o de subjuntivo. Se trata de un tipo de actividad para que refuerce el aprendizaje de la conjugación del presente de subjuntivo, no sólo de los verbos regulares, sino principalmente de los irregulares, al ser este aprendizaje mucho más costoso.

2. "Ejercicio cerrado", es decir, en el que sólo es posible elegir entre una opción correcta. En el caso del presente de subjuntivo para expresar opinión en este tipo de actividades el alumno ya puede trabajar con el contraste puesto que, por ejemplo, tendría que poder seleccionar el tiempo verbal adecuado dentro de una oración como la siguiente: "No creo que puedo/pueda vivir en Madrid durante mucho tiempo". Incluso, si lo que se pretende es reforzar sólo el conocimiento de la forma, se podría buscar la selección entre "No creo que esté/este preparado todavía”. Por tanto, nos encontramos ante las clásicas actividades de metodología estructuralista conocidas como rellena huecos o fill-in-the-gaps exercises. 
3. "Actividades abiertas", son aquellas en las que los alumnos pueden elegir entre diferentes opciones, puesto que entre las que se les ofrecen varias son posibles. Por tanto, esto implica que deben comprender el valor semántico de la construcción y no únicamente la combinación correcta a nivel sintáctico. Por consiguiente, este tipo de actividades requiere un mayor conocimiento lingüístico y comunicativo por parte de los alumnos. En este caso, por ejemplo, se puede tratar de una actividad en la que se formulen preguntas a los alumnos (información que se les proporciona) y ellos deban aportar una respuesta entre diversas posibilidades.

4. "Actividades comunicativas" que permiten al alumno crear su propia respuesta lingüística, es decir, aportan una gran libertad compositiva a los alumnos, fomenta la creatividad y el manejo de la lengua en uso. En estas actividades los alumnos deben trabajar en parejas o grupos para aportar un resultado final. Pensemos, por ejemplo, en la redacción de la crítica sobre el servicio de un hotel o un restaurante o en la elaboración de una conversación informal sobre la valoración de una película de estreno.

5. "Actividades híbridas" son aquellas que contienen elementos de las anteriores, adecuadamente mezclados, por lo que se basan en la combinación de diversas metodologías y permiten adaptarse a los diversos tipos de aprendizaje que desarrollan los alumnos.

\section{RESULTADOS}

\section{El presente de subjuntivo para expresar opinión o valoración en el índice de los manuales}

La introducción al aprendizaje del presente de subjuntivo, tanto a nivel formal, como de uso, se plantea, según el PCIC, a partir del nivel B1 de referencia y se amplía notablemente en el nivel $\mathrm{B} 2^{5}$. En los niveles A1 y A2 este tiempo y modo verbal no se trabaja, porque es necesario que los aprendices adquieran adecuadamente una parte importantes de los tiempos del modo indicativo, así como el imperativo. Por lo tanto, es en los niveles intermedios donde este modo y tiempo verbal se convierte en protagonista de las programaciones.

A continuación, vamos a estudiar cómo se incluye en el índice de los manuales seleccionados los contenidos referidos al presente de subjuntivo y, concretamente, al presente de subjuntivo en los casos en los que este modo y tiempo verbal sirven para dar la opinión o la valoración en español. En primer lugar, se concluye que, en todos los manuales seleccionados, el contenido sobre el presente de subjuntivo y sus funciones se representa en su índice, como se sintetiza en la siguiente tabla, aunque, como se analizará posteriormente de maneras muy diversas:

5 Véase el PCIC: [Enlace] 


\begin{tabular}{|c|c|c|}
\hline Título del manual & $\begin{array}{l}\text { Unidad en la que aparece } \\
\text { el presente de subjuntivo } \\
\text { para expresar opinión o } \\
\text { valoración }\end{array}$ & Presentación del contenido \\
\hline Nuevo español sin fronteras & Unidad 6 & $\begin{array}{l}\text { Funciones comunicativas: expresar prefe- } \\
\text { rencias, gustos, pena, enfado, frustración; } \\
\text { valorar y opinar; expresar sorpresa; expre- } \\
\text { sar aburrimiento. }\end{array}$ \\
\hline Meta ELE B1 & Módulo 6 & $\begin{array}{l}\text { Los verbos de influencia con infinitivo o con } \\
\text { subjuntivo. Las expresiones de opinión y } \\
\text { valoración con indicativo y con subjuntivo. } \\
\text { La acción final del módulo es "Completar } \\
\text { una reclamación y protestar" }\end{array}$ \\
\hline $\begin{array}{l}\text { Eco. Curso modular de español } \\
\text { lengua extranjera }\end{array}$ & Unidad 3 & $\begin{array}{l}\text { Expresar gustos y sentimientos. Oraciones } \\
\text { de relativo. Contraste indicativo/ } \\
\text { subjuntivo. }\end{array}$ \\
\hline Código ELE. B1. & Unidad 5 & $\begin{array}{l}\text { Las oraciones de opinión y valoración con } \\
\text { subjuntivo. }\end{array}$ \\
\hline Español en marcha. 3. & Unidad 6 & $\begin{array}{l}\text { Apartado A: "Opinar. Expresar sentimientos: } \\
\text { me preocupa, me molesta que + subjunti- } \\
\text { vo." Apartado B: "Expresar obligaciones. } \\
\text { Hacer valoraciones: Es conveniente que + } \\
\text { subjuntivo." }\end{array}$ \\
\hline $\begin{array}{l}\text { Método Everest de Español } \\
\text { para extranjeros }\end{array}$ & Lección 5 & $\begin{array}{l}\text { Esquemas gramaticales: La expresión del } \\
\text { mandato, ruego, consejo y deseo. }\end{array}$ \\
\hline Español 2000 & Unidad 19 & $\begin{array}{l}\text { El modo subjuntivo: presente, pret. imper- } \\
\text { fecto, pret. perfecto, pret. pluscuamperf. } \\
\text { Correspondencia de los tiempos del subjun- } \\
\text { tivo con los de indicativo. }\end{array}$ \\
\hline Método 3 & Unidad 6 & $\begin{array}{l}\text { Presente de subjuntivo. Estructuras con } \\
\text { verbos de sentimiento. Estructuras con ver- } \\
\text { bos de pensamiento, sentido y comunica- } \\
\text { ción. }\end{array}$ \\
\hline
\end{tabular}




\begin{tabular}{|l|l|l|}
\hline $\begin{array}{l}\text { Nuevo Sueña 2. Español } \\
\text { Lengua extranjera B1 }\end{array}$ & $\begin{array}{l}\text { Expresar juicios y valoraciones, expresar } \\
\text { acuerdo y desacuerdo total o parcial, mos- } \\
\text { trarse a favor o en contra de una propuesta } \\
\text { o idea, justificar y argumentar una opinión y } \\
\text { expresar certeza }\end{array}$ \\
\hline $\begin{array}{l}\text { Diverso Español B. Curso de } \\
\text { español para jóvenes. }\end{array}$ & Unidad 7 & $\begin{array}{l}\text { Expresar opinión, recomendar, aconsejar, } \\
\text { hablar de los estados de ánimo. }\end{array}$ \\
\hline
\end{tabular}

Tabla 1: Presentación del presente de subjuntivo con valor de opinión y/o en los índices de los manuales seleccionados.

Como se puede deducir de la información contenida en la tabla anterior, la primera conclusión que se extrae es que, en todos los manuales analizados, el presente de subjuntivo para expresa opinión o juicio se incluye en los índices de los manuales. Únicamente hay una excepción en el manual Método Everest de español para extranjeros en cuyo índice no se encuentran referencias a ningún tipo de contenido gramatical, por lo que ni los docentes, ni los discentes, pueden conocer con la lectura del índice qué contenidos gramaticales, léxicos, pragmáticos o culturales van a trabajar en cada lección (terminología empleada en el manual). El índice de este manual es especialmente confuso, aporta escasa información y no aporta información relevante y útil que permita saber qué aporta el estudio de cada una de las lecciones que lo componen. Por lo tanto, salvo esta excepción, en el resto de manuales, es decir, en una abrumadora mayoría, sí se aporta información sobre el presente de subjuntivo para expresar opinión o valoración dentro de los índices.

Sin embargo, es esencial señalar que la presentación en los respectivos índices de este contenido se presenta con grandes diferencias, dependiendo de la metodología mediante la cual se desarrolle el manual. En algunos manuales el contenido se presenta con un enfoque sintáctico, como sucede en los casos de Eco, Código ELE, Método Everest y Español 2000. Frente a esta propuesta, otros manuales con un enfoque más comunicativo, apuestan por presentar el subjuntivo con valor de opinión o valoración en relación a las funciones comunicativas que se pueden desarrollar mediante su empleo. En esta segunda opción se encuentran Meta ELE, Español en marcha, Método 3, Nuevo Sueña 2 y Diverso Español B. Por consiguiente, como hemos indicado, la enunciación del contenido gramatical que estamos analizando está muy condicionada a criterios metodológicos y pedagógicos y no a criterios estrictamente lingüísticos.

\section{Apartados teóricos acerca del presente de subjuntivo con valor de opinión o valoración}

En este epígrafe se van a analizar los contenidos explicativos que se facilitan a los alumnos sobre el presente de subjuntivo con valor de opinión o valoración en los manuales se- 
leccionados. Es decir, se analizará qué explicaciones teóricas se proporcionan en el manual y si éstas detallan adecuadamente las características lingüísticas del presente de subjuntivo para formular la opinión o la valoración desde un prisma pedagógico.

En todos y cada uno de los manuales analizados se aporta una explicación teórica sobre el fenómeno que estamos estudiando. Por lo tanto, se concluye que el estudiante puede acceder a dicha información de manera explícita. A continuación, explicaremos cómo se aborda dicha explicación en cada uno de los manuales:

- Nuevo español sin fronteras: En el apartado "vamos a reflexionar" de la unidad 6 se aporta información léxica sobre los sentimientos. Por ejemplo, se da el sustantivo gusto y la lista de verbos gustar, encantar y entusiasmar. Se aporta una breve explicación gramatical sobre el subjuntivo que se formula mediante una regla fija: $\mathrm{V}(1)+\mathrm{V}(2)$ infinitivo (sujetos iguales): Siento llegar tarde, es que he perdido el autobús. V (1) + que + V (2) subjuntivo (sujetos diferentes): Siento que no puedas venir a la cena. Se incluye un cuadro específico para explicar la expresión de valoraciones que contiene la siguiente información: Ser/estar/parecer + nombresadjetivos + infinitivo / +que + subjuntivo. Se aporta, además, una lista extensa de nombres y adjetivos para que el alumno tenga opciones combinatorias de vocabulario.

- Meta ELE B1: Este manual es plenamente comunicativo y funcional, con una muy escasa presentación de contenidos gramaticales y, éstos se presentan de manera deductiva. Son los alumnos los que extraen sus propias conclusiones sobre las reglas. Al final del manual los autores han incluido un apéndice titulado "Kit de supervivencia" para explicitar contenidos gramaticales y léxicos. Aquí los contenidos se exponen de en modo cuadro ficha. Se encuentra la forma del presente de subjuntivo regular y los verbos irregulares, así como las expresiones para expresar deseo y probabilidad. En este apéndice encontramos la explicación de la expresión del subjuntivo con verbos de influencia (orden, consejo, prohibición, petición...), así como su uso en la valoración: "Es + adjetivo + infinitivo, en general o con el mismo sujeto: Es práctico hablar idiomas. Frente a 'Es + adjetivo + que + subjuntivo'. Particularmente o con sujetos diferentes: Es interesante que sepas arreglar ordenadores." (pág. 79)

- Eco: La expresión del presente de subjuntivo para dar la opinión o hacer valoraciones se encuentra en el apartado "contraste indicativo / subjuntivo" pero no aparece tal cual en el índice. En la sección A de la página 40 se explica el contraste entre los dos modos de la siguiente forma en un cuadro: Indicativo para constatar hechos: que. Es seguro, es evidente, está visto, claro, demostrado que necesitas un médico, frente al uso para hacer valoraciones: Es bueno, malo, mejor, posible, lógico que desprecie la comida porque está enferma. 
- Código ELE: En este manual la explicación de la gramática es muy escasa dentro de las unidades. Apenas se expone la forma del verbo y cuadros muy cerrados sobre la estructura de funcionamiento. El manual presenta un anexo que se desarrolla entre las páginas 135 a 141 con una ampliación de las explicaciones que se han proporcionado en el desarrollo de las unidades. Respecto al presente de subjuntivo para formular la opinión o la valoración lo único que se indica es lo siguiente: Se utiliza un verbo de opinión como pensar, creer, parecer + que + indicativo si es una pregunta o una afirmación. ¿No crees que es muy valiente? Creo que es muy valiente. Se utiliza con subjuntivo si está negado. No creo que sea valiente.

- Español en marcha: Se proporciona una breve explicación mediante un cuadro resumen titulado: "Expresar sentimientos y opiniones". Se aporta la siguiente información: en oraciones dependientes de verbos como gustar, interesar, molestar, preocupar se utiliza infinitivo o subjuntivo. Infinitivo si el sujeto lógico de las dos frases es el mismo. Subjuntivo si el sujeto lógico de las dos frases es diferente.

- Método Everest: La exposición del presente de indicativo para hacer valoraciones o dar la opinión, se encuentra dentro de este manual en la función del subjuntivo para dar consejos. Este manual presenta una página completa en forma de ficha para trabajar el presente de subjuntivo para expresar mandato y ruego, con un recordatorio de la conjugación de dicho tiempo. En Método Everest lo que destaca es que se enfrenta en la misma unidad el uso del presente de subjuntivo con el imperativo, lo que resulta muy poco recomendable pedagógicamente. En otra página completa se incluye una tabla sobre el valor del presente de subjuntivo para rogar, aconsejar y desear. En este cuadro, en la parte dedicada al consejo o las sugerencias, se incluye el presente de subjuntivo para aconsejar, frente a la utilización del presente de indicativo. Se facilitan los siguientes ejemplos: “¿Por qué no + presente de indicativo?" frente a "Aconsejar / sugerir que + subjuntivo".

- Español 2000: Se proporciona una explicación muy escasa sobre el presente de subjuntivo que puede crear confusión en los aprendices y que no hace hincapié en los usos comunicativos de este tiempo. Se explica mediante dos cuadros que se encuentran en la lección 19. En el primer cuadro se aporta la siguiente información: "Expresa la participación subjetiva del hablante. Es el modo de la irrealidad frente al indicativo, que manifiesta la realidad"; "Los tiempos del subjuntivo suelen ir subordinados, integrados en oraciones compuestas" y, por último, "Empleamos el subjuntivo si queremos expresar: duda, deseo, incertidumbre, emociones, sentimientos, ruego, exhortación". Como se puede comprobar, esta explicación del uso es del todo imprecisa, porque todo esto también se puede expresar en indicativo, por lo que no se resuelven las dudas de selección para los aprendices. En el segundo cuadro se aporta la siguiente explicación: "Expresa tiempo presente y futuro"; "Las formas del presente precedidas del adverbio no toman valor de man- 
dato" y "Las formas de primera persona de plural y tercera del singular y del plural se utilizan también como formas del imperativo". Por lo tanto, en este manual se explicita el contraste entre el presente de subjuntivo con otros tiempos y/o modos verbales.

- Método 3: Este manual incluye escasas explicaciones teóricas sobre contenidos gramaticales porque pretende que los aprendices deduzcan las reglas tras estar en contacto con el input que se les facilita. En la unidad 6 se incluye un cuadro en el que el valor del presente de subjuntivo para indicar opinión se pone en contraste con el presente de indicativo mediante la siguiente exposición: "Verbos de pensamiento, sentido y comunicación + que + indicativo. Atención: Si V1 va en forma negativa, entonces V2 va en subjuntivo" (pág. 132). Por tanto, se produce un contraste entre los tiempos, que se explica mediante una regla de aplicación automática.

- Nuevo Sueña 2: En este manual el presente de subjuntivo se presenta en dos cuadros sinópticos. El primero que se titula "Expresar juicios y valoraciones" aporta la siguiente información: ser, estar, parecer + expresión de valoración (necesario, malo, mejor, importante...) Valoración general: verbo indicativo. Infinitivo: Es maravilloso escuchar música en casa. Valoración sobre un sujeto particular: verbo subjuntivo: Es bueno que escuches música relajante. El segundo cuadro se titula "Argumentar una opinión”. Contiene la siguiente información: verbos de lengua, pensamiento y percepción. Verbo principal afirmativo. Verbo sub. indicativo: Creo que es/parece que es/pienso que es/ digo que es... Verbo principal negativo/ verbo subordinado subjuntivo: No creo que sea, no parece que sea...

- Diverso Español B: Las explicaciones gramaticales en este manual, de enfoque muy comunicativo, son escasas. Se aportan en cuadros sinópticos situados en los márgenes de la hoja, lo que tipográficamente ya indica que no se consideran contenidos esenciales por sí mismos. Estos cuadros laterales poseen dos colores: el verde para explicar la parte formal (en este caso la conjugación del presente de subjuntivo) y, el rosa para trabajar las funciones comunicativas. En este caso, respecto a la formulación de la opinión o valoración mediante el presente de subjuntivo únicamente se encuentra la diferenciación en la modalidad oracional: si ésta es afirmativa o interrogativa o si, por el contrario, es negativa. Así se utilizará respectivamente el presente de indicativo o de subjuntivo.

De la exposición anterior, se deduce que las explicaciones teóricas que se aportan sobre el empleo del subjuntivo son escasas, en muchos casos, pueden producir ambigüedad o ser difíciles de asimilar para los aprendices y, en otras, se facilitan reglas exclusivamente combinatorias pero que no fomentan el aprendizaje desde el enfoque cognitivo, es decir, se trabaja el contenido gramatical mediante un aprendizaje memorístico. 
Ejercicios y actividades sobre el presente de subjuntivo para expresar opinión o valoración

En este último apartado del análisis de materiales que se está desarrollando en el presente estudio vamos a realizar una clasificación de las actividades que se presentan en los manuales para que los estudiantes aprendan e interioricen el presente de subjuntivo para expresar la función comunicativa de opinión y/o valoración. Para ello, a continuación, se presenta una tabla sintética con número de actividades de cada tipo que se presentan en cada manual. Tras este cómputo, se podremos establecer unas conclusiones analíticas al respecto:

\begin{tabular}{|c|c|c|c|c|c|}
\hline Título del manual & $\begin{array}{l}\text { Ejercicio } \\
\text { mecánico }\end{array}$ & $\begin{array}{l}\text { Ejercicio } \\
\text { cerrado }\end{array}$ & $\begin{array}{l}\text { Actividades } \\
\text { abiertas }\end{array}$ & $\begin{array}{l}\text { Actividades } \\
\text { comunicativas }\end{array}$ & $\begin{array}{l}\text { Actividades } \\
\text { híbridas }\end{array}$ \\
\hline $\begin{array}{l}\text { Nuevo español sin } \\
\text { fronteras }\end{array}$ & 0 & 3 & 0 & 0 & 0 \\
\hline Meta ELE B1 & 0 & 2 & 1 & 1 & 0 \\
\hline $\begin{array}{l}\text { Eco. Curso modular de } \\
\text { español lengua } \\
\text { extranjera }\end{array}$ & 0 & 3 & 1 & & \\
\hline Código ELE. B1. & 0 & 3 & 0 & 1 & 0 \\
\hline Español en marcha. 3. & 0 & 2 & 0 & 3 & 0 \\
\hline $\begin{array}{l}\text { Método Everest de Es- } \\
\text { pañol para extranjeros }\end{array}$ & 0 & 0 & 2 & 3 & 0 \\
\hline Español 2000 & 0 & 4 & 0 & 0 & 0 \\
\hline Método 3 & 0 & 3 & 2 & 7 & 0 \\
\hline $\begin{array}{l}\text { Nuevo Sueña 2. Español } \\
\text { Lengua extranjera B1 }\end{array}$ & 0 & 0 & 1 & 3 & 0 \\
\hline $\begin{array}{l}\text { Diverso Español B. Curso } \\
\text { de español para jóvenes. }\end{array}$ & 0 & 0 & 1 & 2 & 1 \\
\hline
\end{tabular}

Tabla 2: Análisis cuantitativo de las actividades propuestas en los manuales para trabajar el presente de subjuntivo con valor de opinión y/o valoración. 
A continuación, vamos a indicar el total de actividades computadas, así como el porcentaje sobre el total que pertenece a cada una de las tipologías. De los 49 ejercicios y actividades computados, no se han encontrado ejercicios mecánicos ( $0 \%$ del total), se han presentado 20 ejercicios cerrados y 20 actividades del tipo comunicativo (40,8\% del total) en cada una de las tipologías, se han hallado 8 actividades abiertas $(16,3 \%$ del total) y, por último, una actividad de carácter híbrido (0,49\% del total).

El análisis de estos datos nos permite deducir algunas consideraciones de interés. En primer lugar, resulta llamativo el hecho de no encontrar ejercicios mecánicos. Esto se explica porque en todos los manuales se han incorporado en unidades anteriores a las que se analizan en este estudio. Es decir, nos hemos centrado en estudiar el presente de subjuntivo cuyo uso sea la expresión de valor y/o opinión. Por consiguiente, los ejercicios enfocados al aprendizaje de la morfología verbal se han desarrollado en unidades anteriores a las que estudiamos.

En segundo lugar, es muy significativo que las actividades cerradas (de tipo rellenahuecos) y las actividades comunicativas poseen la misma relevancia y ocupan, cada una de las tipologías, casi la mitad de las actividades propuestas. Esto se puede justificar por dos razones: las actividades cerradas, a día de hoy, pese a la relevancia adquirida por las metodologías activas y/o enfocadas a la acción, siguen siendo necesarias para el dominio de los contenidos gramaticales. Los docentes las necesitan trabajar en sus grupos y los alumnos necesitan interiorizar la gramática mediante este tipo de ejercitación. El hecho de que las actividades comunicativas ocupen casi la otra mitad de las actividades es completamente esperable. Es más, las expectativas iniciales en esta investigación partían de la hipótesis de que el porcentaje de esta tipología de actividades iba a ser todavía su perior. En la actualidad, se ha impuesto el empleo de las metodologías comunicativas (en sus diversas modalidades), por lo que no sorprende que los manuales deban tener en cuenta las necesidades de docentes y discentes.

En tercer lugar, resulta muy significativo que solo se encuentre una actividad de tipo híbrido. Esto implica pensar que todavía las actividades se presentan de manera muy controlada para que el funcionamiento de la actividad dentro del aula resulte de manejo sencillo. No resulta nada extraño que esta actividad híbrida se encuentre en un manual como Diverso, que es el que presenta una metodología más variada y dinámica, algo que se justifica por el tipo de estudiante al que se destina (estudiantes del Bachillerato internacional). Esto estudiantes, cuyo perfil es de excelencia, permiten realizar programaciones dinámicas puesto que pueden afrontarlas tanto por sus capacidades, como por su motivación.

Para concluir hay que señalar que los porcentajes de tipologías de actividades no se reparte de manera homogénea entre los diversos manuales analizados. Cuanto más antiguo en fecha de publicación, como es el caso de Español 2000 y Nuevo español sin fronteras, las actividades cerradas se incrementan de manera sustancial. Es más, en estos dos manuales el $100 \%$ de las actividades pertenecen a esta tipología. Sin embargo, a medida 
que las fechas de publicación se acercan al momento actual y, las metodologías de los manuales se hacen más modernas, las actividades de carácter comunicativo se incrementan. Por consiguiente, se puede concluir que el avance metodológico en la enseñanza de lenguas extranjeras se plantea como uno de los procesos más significativos en el desarrollo de los manuales que se encuentran en el mercado.

\section{CONCLUSIONES}

Después de analizar el tratamiento del presente de subjuntivo con valor de opinión y/o valoración en diez manuales de ELE se obtienen las conclusiones que se exponen a renglón seguido:

- En el 90\% de los manuales analizados, a excepción de Método Everest, se incluye una referencia directa al presente de subjuntivo con valor de opinión y/o valoración en los índices de los manuales. Únicamente en Método Everest esto no ocurre porque, como se ha indicado anteriormente, la exposición inicial de los contenidos resulta muy confusa.

- La presentación en el índice de este contenido es muy heterogénea en los diversos manuales. En el $50 \%$ de los manuales se opta por una presentación basada en un enfoque sintáctico, mientras que en el otro $50 \%$ la propuesta atiende a un enfoque comunicativo. Por lo tanto, podemos encontrar dos tendencias claramente diferenciadas en relación al enfoque metodológico que soportan los respectivos manuales.

- Las explicaciones teóricas que se facilitan dentro de los manuales para que los estudiantes interioricen el presente de subjuntivo con valor de opinión y/o valoración son, en la mayoría de los casos, insuficiente, confusas y basadas en la necesidad de memorización por parte del estudiante.

- Del punto anterior se concluye que sigue predominando un abordaje tradicional, de corte estructuralista de este contenido, que facilita poco la verdadera comprensión de su uso por parte de los estudiantes y, por consiguiente, el empleo en las diversas situaciones comunicativas de manera natural.

- En la totalidad de los manuales se ejercita el empleo del presente de subjuntivo en relación con el contraste en uso del presente de indicativo. Consideramos esto un abordaje correcto porque los estudiantes de ELE presentan la necesidad de resolver la confusión entre los usos que sufren.

- Se ha estudiado el tipo de actividades que se presentan en los manuales y se concluye que ésta es desigual. Priman las actividades cerradas y de tipo comunicativo, realidad que, aunque pueda parecer contradictoria no lo es por las razones que se han explicado en el análisis previo. 
En conclusión, tras analizar los manuales de ELE seleccionados, valorar cómo explican el presente de subjuntivo para enunciar opiniones o valoraciones y qué tipologías de actividades son las más recurrentes en los mismos, podemos determinar que la metodología tradicional sigue teniendo una gran vigencia a la hora de abordar contenidos gramaticales. Esta realidad nos conduce a considerar que es necesario que los conocimientos que actualmente poseemos sobre metodologías activas, se viertan hacia unas propuestas en la didáctica de los contenidos gramaticales más innovadoras. Se debe apostar por llevar al aula materiales más dinámicos, funcionales y comunicativos, para después poder abordar una investigación concluyente en resultados de aprendizaje.

\section{REFERENCIAS}

AMENÓS, J., AHERN, A. y ESCANDELL, Ma V. (2019). Comunicación y cognición en ELE: la perspectiva pragmática, Madrid: Edinumen.

ARTUSI, A. (2020). El tratamiento de los phrasal verbs en los manuales de inglés lengua extranjera, Didácticas específicas, 22, 26-41.

CASTRO, M. (2018). Quería que otros estaban siempre interesados en mí: Un estudio de casos sobre el uso del subjuntivo en aprendices polacos de ELE, Tonos digital: revista de estudios filológicos, 35, 1-23.

CONTRERAS. C. y FERREIRA, A. (2013). Orden de adquisición en español como lengua extranjera: modalidad subjuntiva, Literatura y Lingüística, 28, 249-284.

DE SANTIAGO, J. y FERNÁNDEZ, J. (2017). Fundamentos para la enseñanza del español como 2/L, Madrid: Arco/Libros.

DIDA, B. (2019). Análisis de errores en la adquisición del modo subjuntivo por los estudiantes francófonos de español como lengua extranjera, dir. María del Carmen Miramón-Llorca, Universidad de Alicante, Tesis doctoral.

INSTITUTO CERVANTES (1994). Plan curricular del Instituto Cervantes, Madrid: Instituto Cervantes. En línea: [Enlace].

LLAMAS, J. (2020). El español como lengua extranjera en nativos de lenguas bálticas orientales: análisis de producciones y estudio comparado de las interlenguas españolas de lituanos y letones, dir. Ana María González Martín, Universidad Camilo José Cela, Tesis doctoral.

RODELLAS, C. y RODRÍGUEZ, P. (2018). La adquisición del modo subjuntivo en estudiantes universitarios finlandeses, MarcoELE, 26, 1-22.

TORDERA, J. C. (2016). A vueltas con el subjuntivo en las clases de español como lengua extranjera: subjuntivo y oraciones sustantivas, Foro de Profesores de E/LE, 12, 263-274. 
UGHETTA, G. (2013). Actividades prácticas para la reformulación pragmática y cognitiva del subjuntivo, Mediterráneo. Revista de la Consejería de Educación en Italia, Grecia y Albania, 5, 82-93.

ZU, J. (2018). La adquisición del indicativo y el subjuntivo en estudiantes universitarios chinos, RedELE, 30, 93-130.

Manuales analizados

ALONSO, E., CORPAS, J. y GAMBLUNCH, C. (2016). Diverso Español B. Libro del alumno + cuaderno de ejercicios. Curso de español para jóvenes. Alcobendas, España: SGEL.

CÁRDENAS, F., HIERRO, A. y ROBLES, S. (2013). Método. 3. Madrid, España: AnayaELE.

CASTRO, F., RODERO, I. y SARDINERO, C. (2006). Español en marcha. 3. Alcobendas, España: SGEL.

HERNÁNDEZ, C., HOYOS, C., MENDIZÁBAL, N. y SANZ, B. (2000). Método Everest de español para extranjeros. Nivel intermedio. León: Everest.

SÁNCHEZ, J., MORENO, C. y SANTOS, I. (2005). Nuevo español sin fronteras, B1/B2. Alcobendas, España: SGEL.

RODRÍGUEZ, J. R. y GARCÍA, M. A. (2017). Meta ELE B1. Libro del alumno y cuaderno de ejercicios. Curso de español de supervivencia. Madrid, España: Edelsa.

ROMERO, C y GONZÁlEZ, A. (2005). Eco. Curso modular de Español Lengua Extranjera, Madrid, España: Edelsa.

SÁNCHEZ, J. y GARCÍA, N. (2004). Español 2000, Alcobendas, España: SGEL.

JIMÉNEZ, A., FERNÁNDEZ, J.M. y BASIRICÓ, R. (2017). Código ELE B1. Madrid, España: Edelsa. 